\title{
Assessment of near infrared LED radiation pattern using Otsu thresholding
}

\begin{abstract}
This paper describes the use of Otsu thresholding method in assessing the radiation pattern emitted by near infrared (NIR) LED. The NIR LED configured in this paper is intended to be used as illumination source for the development of a NIR palm vein image acquisition device. The experiment is conducted using a single board computer (SBC) to promote a realtime embedded system development that can be readily integrated as a vein viewing device. Based on the Otsu thresholded image obtained, it is observed that the NIR LED radiation pattern can be accessed subjectively through the thresholding process. The resulted thresholded image can be used as preliminary assessment of the radiation pattern in developing a NIR image acquisition system that fully utilizes the NIR LED properties.
\end{abstract}

Keyword: Near infrared illumination source; Near infrared image acquisition device; Near infrared LED radiation pattern; Otsu thresholding; Palm vein image acquisition; Vein viewing device 